\title{
Matrix Effects on the Proton-beam Induced Reaction of Spirophenanthrooxazine
}

\author{
Min-Seon Seo, Na-Young Kim, and In-Ja Lee ${ }^{*}$ \\ Department of Chemistry, Dongguk University, Gyeongbuk 780-714, Korea. ${ }^{-}$-mail: lia adonggukackr \\ Received October 23, 2009. Accepted November 2. 2009
}

Key Words: Spirophenanthrooxazine. Matrix effects. Proton-beam

Photochromism has been the subject of great interest due to their potential applications in various fields. such as display systems, optical switching and optical memory storage. ${ }^{1: 4}$ Although photochromic dy'es were discovered more than 100 years ago. researches on the compounds and their applications were not widely spread until 1956, when Hirshberg demonstrated photochromic dyes could be applied as an optical memory medium. ${ }^{5}$

Recently. Irie et $\mathrm{al}$. observed that photochromic diarylethene exhibits color change under the $\gamma$-irradiation. ${ }^{6}$ Because its radiation-induced reaction proceeded in a quite similar manner to its photochromic reaction. they proposed its use as the radiation dosimeter. And they predicted photochromic spirocompounds cannot be used for the radiation dosimeter because its colored form is thermodynamically unstable.

However. Moscovitch et al. showed that ionizing radiation such as heavy charged-particles (HCP) is capable of changing the information originally stored within the spiropy ran (SP) doped in polymethyl methacrylate (PMMA), a three-dimensional optical random access memory materials. ${ }^{7 \cdot 11}$ Exposure of SP in PMMA (SP/PMMA) to ionizing radiation resulted in the permanent color change. Depth profiles obtained using a confocal laser scanning microscope prove that the use of SP/ PMMA for real-time dosimeter of neutron or $\mathrm{HCPs}$ is possible.

Very recently. we reported the effects of proton-beam on spirophenanthrooxazine (1.3-dihydro-1.3.3-trimethy l-spiro[2 $\mathrm{H}$ indol-2,3'-[3H]-phenantlur[9, 10-b] $[1,4]$ oxazine]. SPO) dissolved in chloroform ( $\left.\mathrm{SPO} / \mathrm{CHCl}_{3}\right)$. ${ }^{12}$ Under the proton-beam irradiation. SPO permanently decomposes into two main products, 1,3.3-trimethyl-2-oxyindol and phenanthro[9.10-d]oxazole. The reaction is first-order with a rate constant of $0.275 \mathrm{~min}^{-1}$ and proceeds in a different way from its photochromic reaction.

In this study, we report the matrix effects on the proton-beam induced reaction of SPO and propose a new ty pe of color dosimeter based on the reaction. Because the reaction rates drastically vary from matrix to matrix. the results were discussed using stopping power instead of solvent polarity scale.

\section{Experimental Section}

SPO, PMMA, polystyrene (PS) and spectroscopic grade solvents (chloroform and ethanol) were purchased from Aldrich and used as received $4.0 \times 10^{-4} \mathrm{M}$ SPO solutions were prepared by dissolving appropriate amount of SPO in various solvents. SPO doped thin films were prepared on the silicon wafer from the chloroform solutions containing polymer and SPO (3 wt. $\%$ ) by spin coating method. The films were air- dried for 3 days at room temperature and the average film thickness was about $100 \mu \mathrm{m}$.

The liquid samples were irradiated by $25 \mathrm{MeV}$ protonbeam from a MC-50 cyclotron at Korea Institute of Radiological and Medical Sciences (KRAMS). The proton-beam. which exited the cyclotron through the exit window, was directed to the sample located in the atmosphere. The beam current was $10 \mathrm{nA} \sim 1 \mu \mathrm{A}$ and the fluence was adjusted to the range of $1.0 \times$ $10^{161} \sim 1.0 \times 10^{14}$ ions $/ \mathrm{cm}^{2}$ by changing the irradiation time.

The polymer films were irradiated either by $25 \mathrm{MeV}$ proton beam from the cyclotron or by $3.4 \mathrm{MeV}$ proton beam from a Tandem accelerator. In the Tandem accelerator at Korea Institute of Geoscience and Mineral Resources (KIGAM). the irradiations were performed by beam scanning under vacuum at $100 \mathrm{nA}$ or $1 \mu \mathrm{A}$ and the fluence was $1.0 \times 10^{12} \sim 4.0 \times 10^{1+}$ ions $/ \mathrm{cm}^{2}$

The UV-Vis spectra were measured using a Cary 4000 spectrophotometer (Varian). For liquid sanples, a cell with appropriate path lengths $(2.5$ and $10 \mathrm{~mm}$ ) was selected by considering absorbance in the visible region and then the difference in the path lengths was corrected to give overlaid spectra.

SRIM $2003^{13}$ was utilized to calculate stopping power and range of ions in matter. In the calculations. energy loss by the sample vial was taken into account for liquid samples.

\section{Results and Discussion}

SPO is a photochromic and thermochromic compound. ${ }^{2.3 .14}$ Under UV radiation. SPO experiences a photochenucal transformation to its colored photomerocyanine form (PMC), which reverts back to SPO in the dark (Scheme 1).

Figure 1 is a plot depicting the effects of proton fluence on the absorption spectra of $\mathrm{SPO} / \mathrm{CHCl}_{3}$ solution. Before the irradiation, the solution shows an absorption band at $350 \mathrm{~nm}$

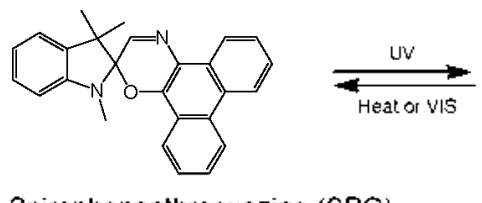

Spirophenanthrooxazine (SPO) closed form

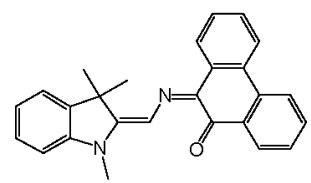

Photomerocyanine (PMC) open form
Scheme 1. Photochromism of SPO 


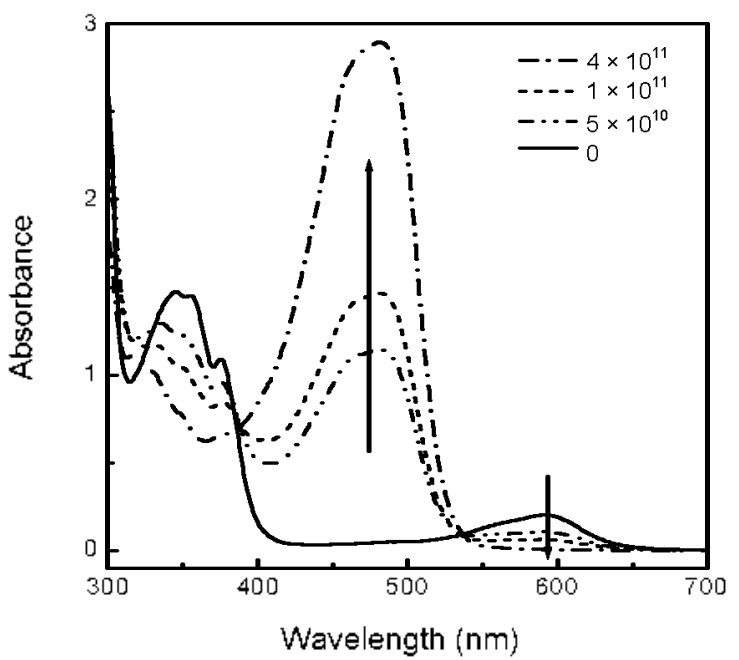

Figure 1. Effects of proton fluence on the spectra of $\mathrm{SPO} / \mathrm{CHCl}_{3}$. The ion energy was $25 \mathrm{MeV}$ and the numbers in the figure indicate ion fluence in the unit of ions $/ \mathrm{cmin}^{2}$.

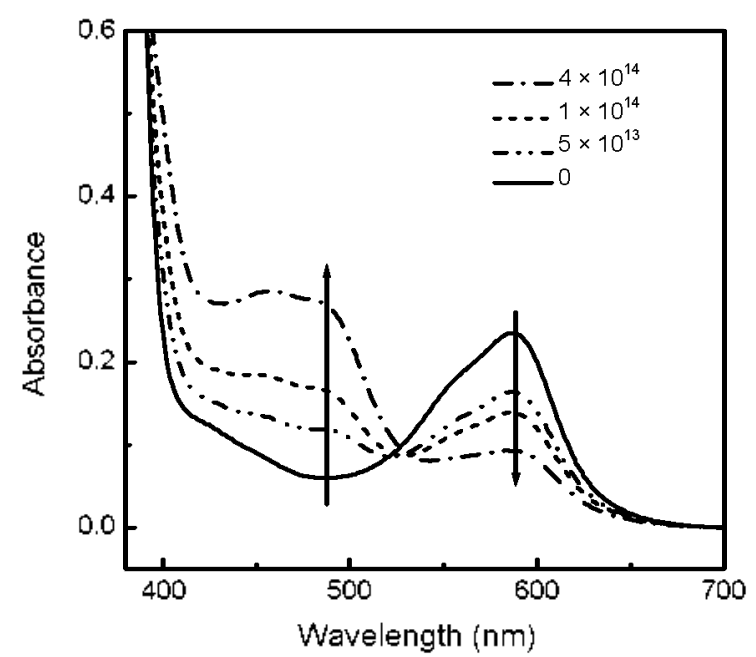

Figure 2. Effects of proton fluence on the spectra of SPO/PS. The ion energv was $25 \mathrm{MeV}$ and he numbers in the figure indicate ion fluence in the unit of ions $/ \mathrm{cmin}^{2}$.

and a smaller one at $590 \mathrm{~nm}$. which corresponds to PMC produced by thermochromic reaction. Upon the proton irradiation $(25 \mathrm{MeV})$. a new band grows at $480 \mathrm{~nm}$, while the bands at $350 \mathrm{~nm}$ and at $590 \mathrm{~nm}$ concomitantly decrease. The band at $480 \mathrm{~nm}$ rapidly grows with the further irradiation and maximized at the fluence of $4 \times 10^{11}$ ions $/ \mathrm{cm}^{2}$. In the previous pape. ${ }^{13}$ we interpreted this results as a proton-beam induced decomposition reaction. which is different from photochromic reaction. Because $\mathrm{PMC}$ is in equilibrium with $\mathrm{SPO}$. decomposition of $\mathrm{SPO}$ accompanies with reduction in PMC concentration and, therefore, results in simultaneous decrease in both bands. The species absorbing at $480 \mathrm{~nm}$. we referred it as " $\mathrm{B}$ ", was attributed to a minor product possessing extended conjugated double bonds.

Figure 2 represents the change in the absorption spectra of $\mathrm{SPO}$ in $\mathrm{PS}$ (SPO/PS) under $25 \mathrm{MeV}$ proton beams. Trends in

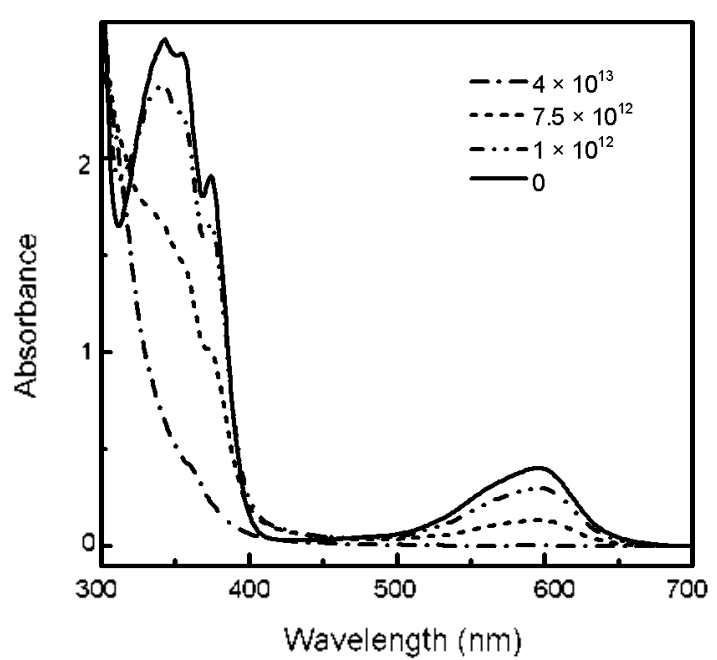

Figure 3. Effects of proton fluence on the spectra of SPO/ethanol. The ion energy was $25 \mathrm{MeV}$ and the numbers in the figure indicate ion fluence in the unit of ions/ $\mathrm{cm}^{2}$.

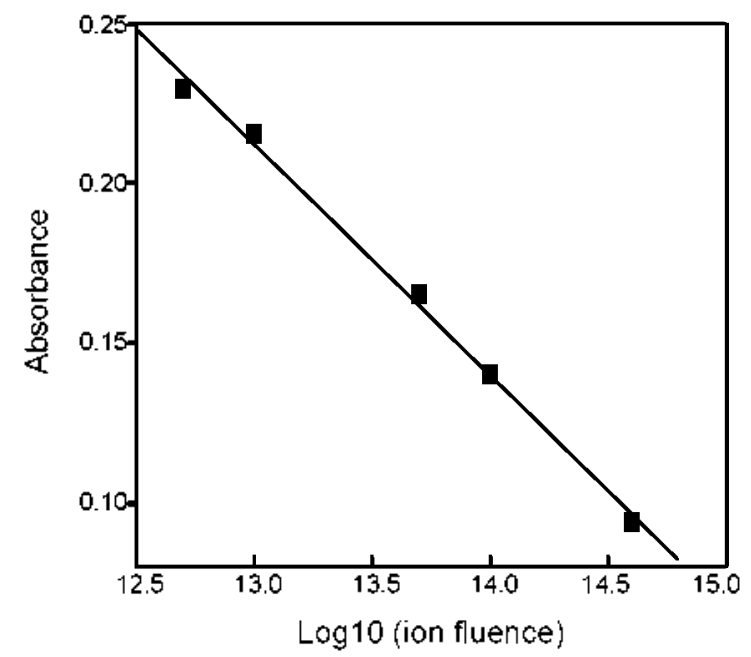

Figure 4. Proton fluence response curve of absorbance at $590 \mathrm{~nm}$ for SPO/PS. The proton energy was $25 \mathrm{MeV}$.

the spectral changes are very similar to those of $\mathrm{SPO} / \mathrm{CHCl}_{\bar{s}}$ except fluence at which $470 \mathrm{~nm}$-band starts growing. The fluence is about $5 \times 10^{12}$ ions $/ \mathrm{cm}^{2}$, while it is as low as $1.0 \times$ $10^{10}$ ions $/ \mathrm{cm}^{2}$ in $\mathrm{SPO} / \mathrm{CHCl}_{3}$.

Under $3.4 \mathrm{MeV}$ proton beams, SPO/PS and SPO/PMMA behave similarly to Figure $I$ and 2 except onset fluence of 470 nm-band. The fluence is $5 \times 10^{12}$ ions $/ \mathrm{cm}^{2}$ and $2.5 \times 10^{11}$ ions/ $\mathrm{cm}^{2}$. respectively. These results show that in PS matrix, the reaction under $3.4 \mathrm{MeV}$ beams is about 10 times faster than under $25 \mathrm{MeV}$ beams and that the rate is slightly faster in PMMA than in PS if the ion energy is same. The results seem to indicate that proton-beam induced reaction proceeds in the same mechanism but at different rate in chloroform. PS and PMMA environments.

In ethanol (Figure 3), there is only a slight decrease in the absorbance throughout the whole spectral range even at the 
fluence of about $4 \times 10^{11}$ ions $/ \mathrm{cm}^{2}$, at which SPO completely decomposed in chloroform. ${ }^{\text {I2 }}$ It was not until the fluence reaches $+\times 10^{13}$ ions $/ \mathrm{cm}^{2}$. which is about 100 times higher than in clloroform solution. that SPO completely decomposes. However there is no indication of $\mathrm{B}$ production. Because in chloroform the molar absorption coefficient of $B$ at $480 \mathrm{~nm}$ seems to be greater than that of SPO at $350 \mathrm{~nm}$. it is expected that B will be easily observed if there is any. However, $B$ is not traced even 1 month after terminating the irradiation. This fact seems to support that B cannot be formed in ethanol. not that its formation rate is too slow:

Solvent effects on organic reactions are usually explained using solvent polarity. ${ }^{15}$ Shin et al. reported that $\pi^{*}$-scale well explains the solvent effects on the thermal decay reaction of PMC form of spironaphthooxazine (SNO) produced by laser flash photolysis method. ${ }^{16}$ SNO has one phenyl ring less on oxazine moiety than SPO and experiences photochromic and thermochromic reaction in a similar manner to SPO. They showed that the thermal bleaching reaction of PMC is operative in a single mechanism in various solvents although the kinetic behavior of the reactions is classified into three classes. polychlorinated aliphatic solvents. alcoholic solvents and nonchlorinated solvents. Chloroform (0.58) and ethanol (0.54) are pretty much the same in $\pi^{-}$value ${ }^{1 /}$ and the reaction in clloroform was about two times faster than in ethanol. ${ }^{16}$ The ratio of the highest- to lowest-rate constant among twelve solvents is about 10 at the most.

However. things are quite different in the ion-beam induced reaction. The ion-beam induced reaction in clloroform is about 40 times faster than in ethanol and about 1000 times faster than in PS. Such a big difference in the reaction rate between two solvents cannot be properly explained using solvent polarity. Furthermore. the fact that the reaction rate of SPO in PS depends on the ion energy cannot be explained by the solvent polarity. Instead. it seems to be closely connected with stopping power. that is the interaction of the ionizing radiation and the matter. When ion beams traveling through materials. charged particles ionize atoms or molecules which they collide and. therefore gradually lose their energy in many steps. Stopping power means the average energy loss of each particle per unit path length and is expressed: $S(E)=-\frac{d E}{d x}$ where $E$ is energy' and $x$ is the path length. In particular, the polymer within the penetration depth will experience chain scission or cross linking and transform to other molecular structure when collide with energetic ions. In the present paper. their contribution to the spectral change in visible region is assumed to be negligible because under the given condition the absorbance change of SPO is drastic.

We observed that no matter whether proton energy is 3.4 MeV or $25 \mathrm{MeV}$. PMMA and SPO/PMMA films melt if ion current is $1.0 \mu \mathrm{A}$ and if ion fluence is higher than $5 \times 10^{14}$ ions/ $\mathrm{cm}^{2}$. However, those films do not melt under the same condition but the ion current of 100 nA or less. Meanwhile. PS and SPO/ PS do not melt under any of above conditions. Because the melting points of PS and PMMA are 240 and $130-140^{\circ} \mathrm{C}$. respectively, it is deduced that the temperature of PMMA films during irradiation is higher than at least $130^{\circ} \mathrm{C}$ at the ion current of $1.0 \mu \mathrm{A}$ and at the ion fluence of $5 \times 10^{14}$ ions $/ \mathrm{cm}^{2}$. The facts that both doped and undoped PMMA films melt at same condition and SPO content is only $3 \mathrm{wt} \%$ in the film imply that the melting is mainly caused by energy absorbed by PMMA matrix itself. Moscovitch et al. calculated that the temperature along each HCP track far exceeds $300^{\circ} \mathrm{C}$ in PMMA. These results may imply that the decomposition reaction of SPO proceed either thermally or radiationally.

According to SRIM calculation, the Bragg peak of PS is located at $174 \mu \mathrm{m}$ and $6.0 \mathrm{~mm}$ for 3.4 and $25 \mathrm{MeV}$ protons. respectively, and stopping power is 1.4 and $0.23 \mathrm{eV} / \mathrm{A}$. respectively. Because the film thickness is about $100 \mu \mathrm{m}$. it is easily seen that both $3 .+\mathrm{MeV}$ and $25 \mathrm{MeV}$ ions pass through the matrix. The fact that stopping power for $3.4 \mathrm{MeV}$ proton is about 6 times higher than that for $25 \mathrm{MeV}$ proton implies that the energy absorbed from $3.4 \mathrm{MeV}$ proton is about 6 times more than that from $25 \mathrm{MeV}$ proton. This agrees well with the experimental result that the reaction under $3.4 \mathrm{MeV}$ beants is about 10 times faster than that under $25 \mathrm{MeV}$.

For 3.4 MeV protons, the stopping power of PMMA and PS are calculated to be 1.58 and $1.4 \mathrm{eV} / \mathrm{A}$, respectively. Because PMMA has higher stopping power than PS. more energy' will be deposited to PMMA than to PS for same charged particle. This result predicts that the proton-beam induced reaction is slightly faster in PMMA than in PS and the prediction agrees with the experimental result mentioned earlier.

For color dosimeter application, it is needed to show a linear relationship between the ion fluence and the radiation induced absorbance change. Figure 4 shows that the absorbance of SPO/ PS at $590 \mathrm{~nm}$ linearly decreases with the proton fluence. The linear relationship indicates that the SPO/PS can be applied to a color dosimeter. Because the radiation induced reaction of SPO is permanent. the film is not reusable.

In summary. the rate of the proton-beam induced reaction of SPO depends on the ion energy and greatly influenced by matrix. The results are qualitatively explained in terms of stopping power and showed that SPO has the potential application in a new type of color dosimeter based on the reaction.

Acknowledgments. Author would like to acknowledge Miss T.-W. Kang for sample preparation.

\section{Refeiences}

1. Hirshberg, Y. J. Am. Chem. Soc 1956, 68, 2304

2. Photochromism: Molecules and Sustems; Dutr, H.: Bouas-Laurent, H., Eds; Elsevier: New York, 1990.

3. Berthelson, R. C. In Techniques of Chentistr: Photochrontisnt, Brown, C. H., Ed.: Wiley-Interscience: New York, 1971: Vol. 3.

4. Dür. H. Angew. Chem., Int. Ed. Engl. 1989. 28.413.

5. Chu, N. Y. C. Can. J. Chem. 1983, 61, 300 .

6. Irie, S.; Kim, M.-S.; Kawai, T.; Irie, M. Bull Chem. Soc. Jpn. 2004, 77,1037

7. Moscovitch, M.; Emfietzoglou, D. J. Appl. Phus. 1997, 81(1), 58.

8. Moscovitch M.: Phillips, G. W. Micl Instr. Meth Phus Res. B $2001,18+, 207$

9. Moscovitch, M. L.S. patent 5319210,1994

10. Moscovitch, M. LiS. patent 5498876,1996

11. Moscovitch, M.: Phillips, G. W.: Cullum. B. M.: Mobley, I.: Bogard, J. S.; Entietzoglou, D.; Vo-Dinh, T. Rodiation Protection 
Dosimetn 2002,10(1-4), 17

12. Lee, I-J.; Kan1, T.-W, Kin, T.-E. Chem. Lett. 2008, 37(11), 1154.

13. Ziegler, T. F. Manoyan, I. M. Kucl Instr and Meth. Ih Phys. Res. $B$ 1988, 35, 215. Computer program and additional information are available at http:/ $/$ ww srim.org.

14. Lee, D.-H.: Lee, M.: Chung, C : Lee, W.-H.: Lee, I.-T. Chem Lett. 2003, 32(7), 578 .
15. Reichardt, C. Solvents and Solvent Effects in Organic Chemistry. VCH: Weinheim, 1988.

16. Shin, T.-W.: Cho, Y.-S.; Huh, Y.-D.; Lee, K.-D; Yang, W.; Park, T; Lee, I.-J. J. Photochent. Photobiol A: Chem. 2000, 137, 163.

17. Kamlet, M. J.: Abboud, J. L. M:; Abraham, M. H.: Taft, R. W.J. Org. Chem. 1983, $48,2877$. 